\title{
Multi-Channel Sound Synthesis System in Open Area. A Case Study with the Use of FEM
}

\author{
W. Ciesielka* And R. Filipek \\ AGH University of Science and Technology, Al. A. Mickiewicza 30, 30-059 Krakow, Poland
}

\begin{abstract}
This paper presents a new model of the multi-channel sound synthesis system. The research objective was to design and construct a sixteen-channel sound control system located in an open area with dimensions of $23.48 \mathrm{~m} \times$ $17.48 \mathrm{~m}$. The main goal of the system operation is to provide, for the controlled acoustic field, the uniformity of the parameters distribution while minimizing the sound pressure level in the protected area. The modeling and simulation researches have been performed using the Finite Element Method (FEM). The results of the analysis include distributions of the sound pressure levels and phase angle for the selected frequencies. The paper presents results of sensitivity studies and optimization for different variants of the sound synthesis system.
\end{abstract}

DOI: $10.12693 /$ APhysPolA.125.A-88

PACS: 43.50.Ki, 43.55.Jz, 43.55.Ka, 43.60.+d

\section{Introduction}

Sound synthesis is a set of procedures for signal generation and processing of these signals and the input information in order to produce at the output of the synthesis system (synthesizer) an acoustic signal with assumed frequency and time parameters [1]. Different sound synthesis methods identified in literature of the subject include five groups: special sound synthesis, speech synthesis, music synthesis, sound sources synthesis [1-6], and the synthesis of sounds in the sound field [1, 2, 6-8]. In the synthesis of sound in the acoustic field direct methods can be used, i.e. measurement methods and digital signal processing $[1,2,6,8]$ as well as computational methods which include: geometric methods $[7,9,10]$, finite element methods (FEM) [8, 10], and boundary element methods (BEM) [10, 11]. This paper will present sensitivity analysis and optimization of multi-channel sound synthesis system in the open area with the use of FEM.

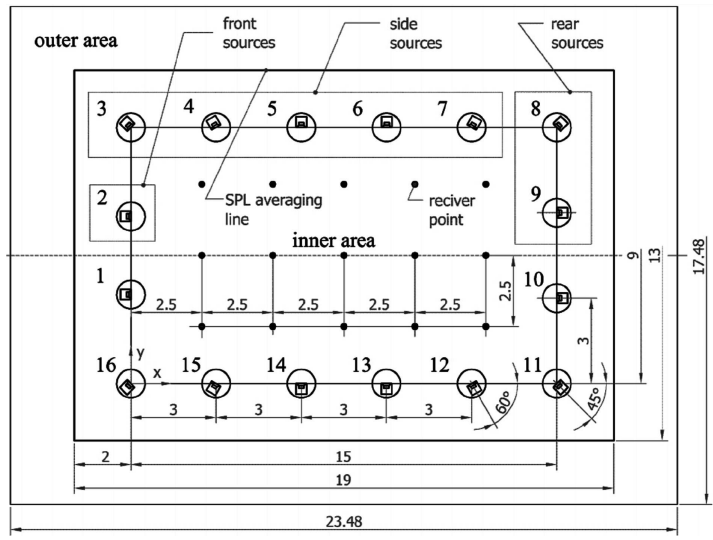

Fig. 1. Geometry of the model with the receiver points and the averaging line location.

*corresponding author; e-mail: ghciesie@cyf-kr.edu.pl
The aim of the study is to determine the optimal parameters of the system due to the assumed quality index which includes minimization of sound pressure levels outside the controlled area and uniformity of the sound field within the area (Fig. 1).

\section{Sound field model}

The object of the study was an open space area where the loudspeakers are located on perimeter of a rectangle measuring $15 \mathrm{~m} \times 9 \mathrm{~m}$. To solve the lossless wave equation [12] FEM [10, 13] was applied. After completion of the discretization process using Galerkin's method, the Helmholtz equation

$$
\frac{\omega^{2}}{c^{2}} \boldsymbol{P}+\nabla^{2} \boldsymbol{P}=0
$$

where $\boldsymbol{P}$ is the acoustic pressure, $\omega$ is the angular frequency, $c$ is the speed of sound, finally leads to the discretized wave equation:

$$
\left(\omega^{2}\left[M_{e}^{P}\right]+\left[K_{e}^{P}\right]\right)\left\{P_{e}\right\}=0,
$$

where $\left[M_{e}^{P}\right],\left[K_{e}^{P}\right]$ are matrixes of the medium's mass and stiffness, respectively, and $\left\{P_{e}\right\}$ is the nodal acoustic pressure vector. The analysis was carried out for a steady state system and the harmonic excitation and response in center frequencies of one-third-octaves in a range from $63 \mathrm{~Hz}$ to $250 \mathrm{~Hz}$. The medium through which acoustic waves propagate was the atmospheric air for which the speed of sound of $343 \mathrm{~m} \mathrm{~s}^{-1}$ and the density of $1.2 \mathrm{~kg} \mathrm{~m}^{-3}$ was assumed. In the presented model, the sound source was implemented by defining the Dirichlet boundary condition on the spherical surface surrounding the source, i.e. setting values of the complex sound pressure at the boundary of the acoustic medium. Detailed source modeling process has been described by the authors in $[8,3,4]$ and the directional characteristics of the used sound source are shown in Fig. 2. The main step in the modeling process was to develop a model of the acoustic field in an open area segment with dimensions of $23.48 \mathrm{~m} \times 17.48 \mathrm{~m} \times 2 \mathrm{~m}$. The finite element model was developed and further calculations performed in ANSYS engineering package [15]. For the discretization of the 


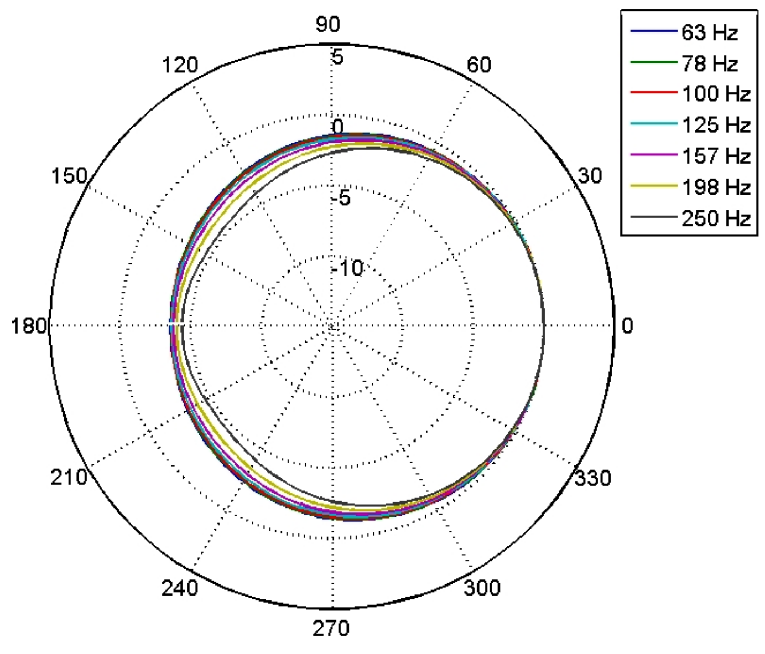

Fig. 2. Sound source horizontal directivity patterns.

acoustic volume, twenty and eight-node Fluid220 type elements were used. Most of the mesh consisted of brick elements, however, in the area near the sources, due the geometrical irregularities, it was necessary to use the tetrahedral element option. The total emission of the acoustic energy from the system was achieved by defining on the acoustic medium boundary perfectly matched layer (PML) element layers [13]. The study adopted the PML layer thickness of $4.29 \mathrm{~m}$. The model contained approximately 700 thousand nodes and 200 thousand elements. On the surface intersecting sources in a symmetrical manner, the symmetry boundary condition (total reflection of acoustic waves from perfectly rigid surface) was defined. Distribution of the sound pressure level for seven frequencies and sixteen operating sources was calculated by addition of distributions received in sixteen subsequent partial calculations for sources operating individually. In every step of the calculation the volume inside the sphere surrounding the operating source was adopted as not filled, and for other sources it was assumed to be filled with an acoustic medium.

\section{Sensitivity study and optimization}

The next step in this study was to determine the sensitivity of systems output parameters to input parameters and optimize the multi-channel sound synthesis system in the open area. The study utilized the design of experiments (DOE) method [14] using a hybrid plan combining optimal-space-filling with an extended central composite design plan. In experiments designed to study the sensitivity Spearman's rank correlation coefficient was used. The system optimization was performed with the Screening method.

Equation (3) describes the Spearman correlation coefficient which represents the measure of the strength of the monotonic relationship, not necessarily linear, between system input and output. It has been defined for each interaction between two sets of observation parameters $x_{i}$ and $y_{i}$ as

$$
r_{s}=\frac{\sum_{i=1}^{n}\left(R_{i}-\bar{R}\right)\left(S_{i}-\bar{S}\right)}{\sqrt{\sum_{i=1}^{n}\left(R_{i}-\bar{R}\right)^{2}} \sqrt{\sum_{i=1}^{n}\left(S_{i}-\bar{S}\right)^{2}}},
$$

where $R_{i}$ is the rank of $x_{i}$ within the set of observations $\left[x_{1} x_{2} \ldots x_{n}\right]^{T}, S_{i}$ is the rank of $y_{i}$ within the set of observations $\left[y_{1} y_{2} \ldots y_{n}\right]^{T}, \bar{R}, \bar{S}$ are the average ranks of a $R_{i}$ and $S_{i}$ respectively.

TABLE I

Sound sources parameters. Sound power level $[\mathrm{dB}]-(1)$, time delay $[\mathrm{ms}]-(2)$.

\begin{tabular}{|c|c|c|c|c|c|c|c|c|c|c|c|}
\hline \multirow{3}{*}{ Group } & \multirow{3}{*}{$\begin{array}{l}\text { Pair } \\
\text { No. }\end{array}$} & \multicolumn{6}{|c|}{ Sound source } & \multicolumn{4}{|c|}{ Variant } \\
\hline & & \multirow{2}{*}{ No. } & \multicolumn{2}{|c|}{ Coordinates } & \multirow{2}{*}{ No. } & \multicolumn{2}{|c|}{ Coordinates } & \multicolumn{2}{|c|}{0} & \multicolumn{2}{|c|}{1} \\
\hline & & & $\mathrm{x}[\mathrm{m}]$ & $\mathrm{y}[\mathrm{m}]$ & & $\mathrm{x}[\mathrm{m}]$ & $\mathrm{y}[\mathrm{m}]$ & (1) & $(2)$ & (1) & $(2)$ \\
\hline front & 1 & 1 & 0 & 3 & 2 & 0 & 6 & 100 & 0 & 100 & 0 \\
\hline \multirow{5}{*}{ side } & 2 & 3 & 0 & 9 & 16 & 0 & 0 & 100 & 0 & 100 & 0 \\
\hline & 3 & 4 & 3 & 9 & 15 & 3 & 0 & 100 & 0 & 100 & 9 \\
\hline & 4 & 5 & 6 & 9 & 14 & 6 & 0 & 100 & 0 & 100 & 18 \\
\hline & 5 & 6 & 9 & 9 & 13 & 9 & 0 & 100 & 0 & 100 & 28 \\
\hline & 6 & 7 & 12 & 9 & 12 & 12 & 0 & 100 & 0 & 100 & 38 \\
\hline \multirow{2}{*}{ rear } & 7 & 8 & 15 & 9 & 11 & 15 & 0 & 100 & 0 & 100 & 47 \\
\hline & 8 & 9 & 15 & 6 & 10 & 15 & 3 & 100 & 0 & 100 & 47 \\
\hline
\end{tabular}

Design parameters ranges and obtained sound field parameters.

TABLE II

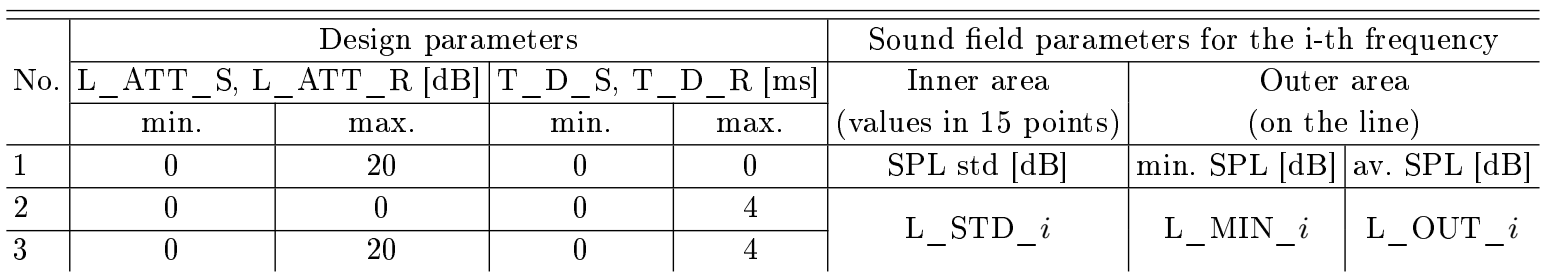


As a part of the work, three system variants were analyzed. Sound source parameters for every variant are summarized in Table I and decision and output parameters are given in Table II. As the decision parameters, sound source parameters grouped into the side and rear sources (Figure 1) were selected. The attenuation level for side sources ( $\mathrm{L}_{-} \mathrm{ATT} \mathrm{AT}_{-} \mathrm{S}$ ) and rear sources ( L ATT R) was modified in the range from $0 \mathrm{~dB}$ to $20{ }^{-} \mathrm{dB}$ and the time delay for side sources ( $\mathrm{T}_{-} \mathrm{D}_{-} \mathrm{S}$ ) and rear sources $\left(T_{-} D_{-} R\right)$ in the range from $\overline{0}$ to 4 ms. Output parameters were determined for every $i$-th of seven one-third-octave center frequencies in the range from $63 \mathrm{~Hz}$ o $250 \mathrm{~Hz}$. In the inner area, the standard deviation L_STD_ $i$ and the minimum sound pressure level $\mathrm{L}_{-} \mathrm{MIN}_{-} \bar{i}$ at 15 points were computed. In the outer area, the average sound pressure level L_OUT_ $i$ along a line at the distance of $2 \mathrm{~m}$ from the loudspeakers was calculated. Then, the objective function $J$ was defined (4) as the sum of terms for the outer area $J_{\mathrm{OUT}}(5)$ and the inner area $J_{\mathrm{STD}}(6)$ :

$$
\begin{aligned}
& J=J_{\mathrm{OUT}}+J_{\mathrm{STD}} \\
& J_{\mathrm{OUT}}=\sum_{i=1}^{7}\left(10 \log _{10}\left(\frac{1}{N} \sum_{k=1}^{N} \frac{p_{i k}^{2}}{p_{0}^{2}}\right)\right), \\
& J_{\mathrm{STD}}=\sum_{i=1}^{7}\left[\frac { 1 } { 1 5 } \sum _ { j = 1 } ^ { 1 5 } \left(10 \log _{10}\left(\frac{p_{i j}^{2}}{p_{0}^{2}}\right)\right.\right. \\
& \left.\left.-\frac{1}{15} \sum_{l=1}^{15} 10 \log _{10}\left(\frac{p_{i l}^{2}}{p_{0}^{2}}\right)\right)^{2}\right]^{-\frac{1}{2}},
\end{aligned}
$$

where $p_{i k}, p_{i j}, p_{i l}$ are sound pressures for $i$-th frequency and $k$-th node on the line and either $j$-th or $l$-th point, $p=2 \times 10^{-5} \mathrm{~Pa}$ (reference pressure) and $N$ is the number of nodes on the line.

The optimization process also includes additional criterion (7) describing the condition to provide a minimum sound pressure level in each of the 15 points for each of the seven analyzed frequencies:

$$
\begin{gathered}
\mathrm{L}_{-} \mathrm{MIN}_{-} i=\min \left(10 \log _{10}\left(\frac{p_{i j}^{2}}{p_{0}^{2}}\right)\right) \geq 70[\mathrm{~dB}], \\
i=1 \ldots 7, j=1 \ldots 15 .
\end{gathered}
$$

In order to perform optimization the Screening method was chosen, which is non-iterative method based on the direct sampling and quasirandom Hammerslay's number generator. The set of pseudorandom points included 6000 samples. Maximum of 3 candidate solutions have been obtained and verified. The decision support process included minimizing the objective function (4) and the criterion (7).

Figure 3 presents the system sensitivity represented by the correlation coefficient for the variant 0 and the variant 1 for the modification of attenuation levels, time delays and the combination of attenuation levels and time delays. The sensitivity analysis of the results showed that impact of the attenuation of sound sources power level on

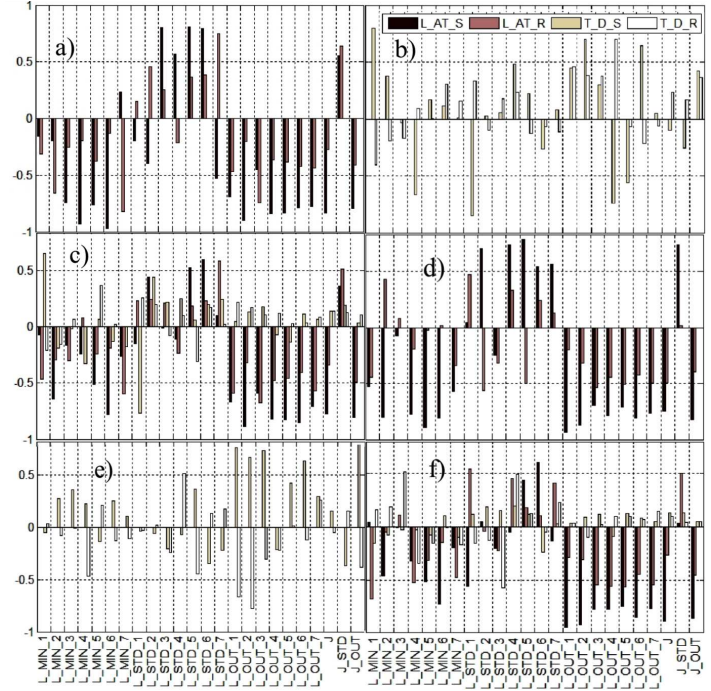

Fig. 3. System sensitivities for variant 0 (up) and variant 1 (down) for: (a) and (d) - attenuation levels; (b) and (e) - time delays, (c) and (f) — both attenuation levels and delays.

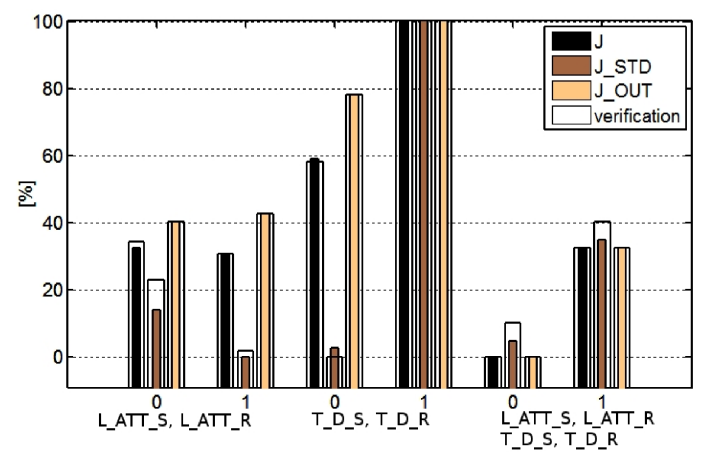

Fig. 4. Normalized objective function and its components.

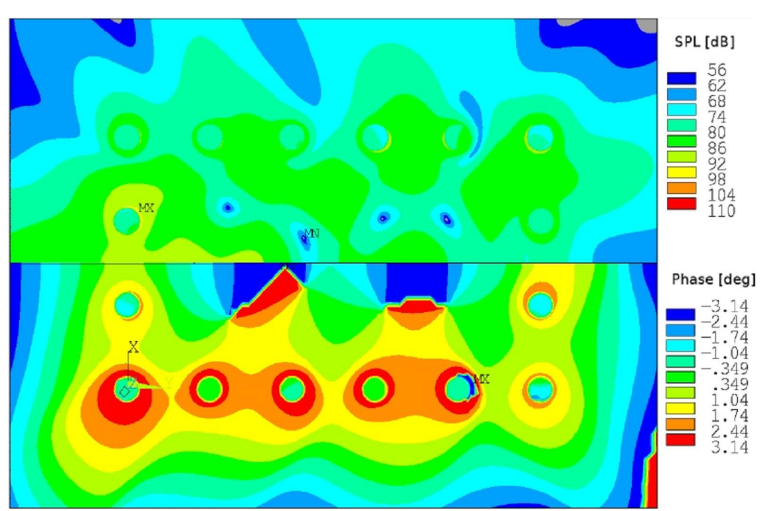

Fig. 5. SPL distribution (up) and phase angle (down) for the best solution and $63 \mathrm{~Hz}$ frequency. 

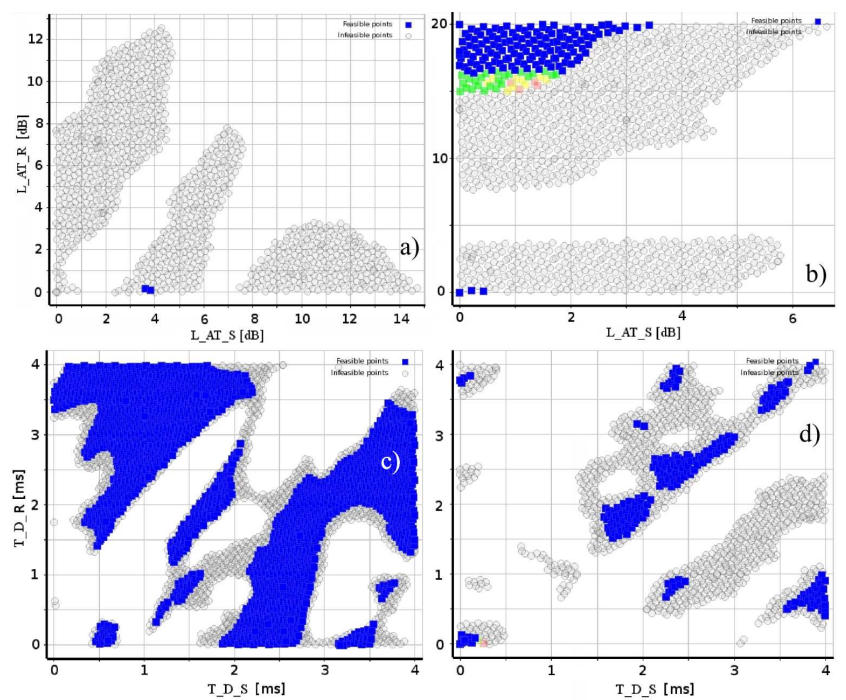

Fig. 6. Pareto fronts for modification of attenuation levels only or time delays only: (a) variant 0 , attenuation levels; (b) variant 1 , attenuation levels; (c) variant 0 , delays, (d) variant 1 , delays.
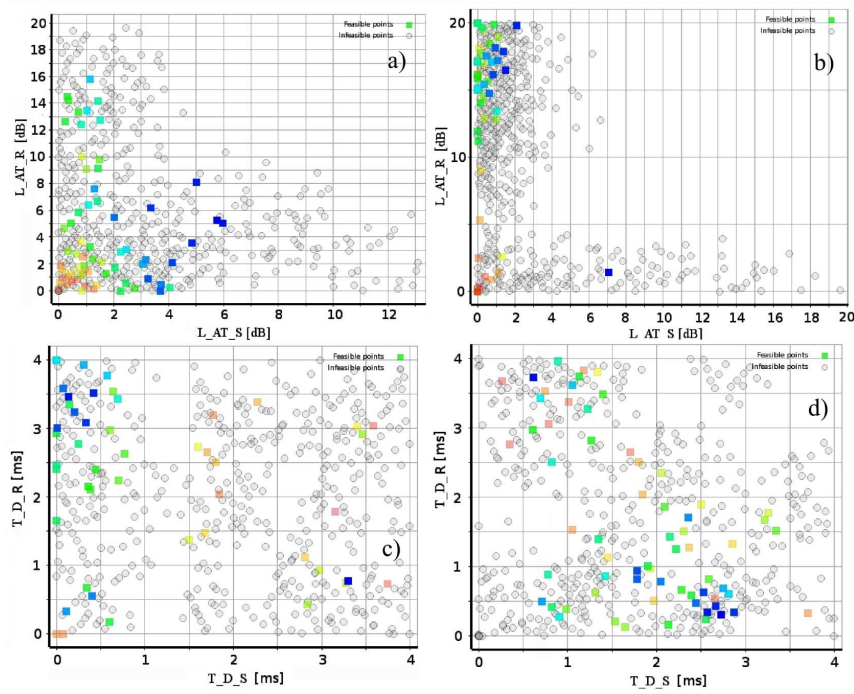

Fig. 7. Pareto fronts for modification of attenuation levels and time delays simultaneously: (a) variant 0 , attenuation levels; (b) variant 1, attenuation levels; (c) variant 0 , delays; $(\mathrm{d})$ variant 1 , delays. the sound field parameters was greater than this of the introduced time delay. The standard deviation of the sound pressure level at points in the inner area shows that attenuation levels and delays effect are highly variable. Figure 4 shows the normalized objective function and its components for optimal solutions for the above variants and 1 with modification of attenuation levels, delays and both attenuation levels and delays. The bars indicate the percentage difference between different variants $(0 \%$ is the best option, $100 \%$ is the worst option for the objective function and its corresponding components). On the graph verified values are marked with the transparent frame. Figure 5 presents distribution of sound pressure levels and phase angle for the best solution and $63 \mathrm{~Hz}$ frequency. Detailed results obtained in the optimization process are presented in Figs. 6 and 7 showing the set of solutions in the form of Pareto fronts. Table III lists parameters for the best three solutions and different variants and Table IV - the sound field parameters obtained for the best solution.

\section{Summary}

The paper presents original research model of the multi-channel sound synthesis system developed with the use of FEM. As a part of the work sensitivity testing and optimization for various combinations of the sound synthesis located in an open area segment have been carried out. The sensitivity analysis of the results for all tested variants revealed that the effect of attenuation of sound sources power level on the sound field parameters used for the synthesis was more significant than the introduced time delay.

The use of Screening methods in the optimization process was helpful in identifying the best solutions for different variants of sound synthesis systems. As a part of the work, the methodology of sensitivity analysis and optimization of multi-channel sound synthesis system in an open area segment have been developed.

The results allow the parameters tuning to optimize multi-channel sound field synthesis in the low frequency range. Their inclusion in parallel with geometrical methods and implementing on the card with a digital signal processor [7] allow to control the sound according to the given criteria in the full audio frequency range

The first three best solutions of the optimization process.

TABLE III

\begin{tabular}{|c|c|c|c|c|c|c|c|c|c|c|c|c|c|c|c|c|c|c|}
\hline No. & \multicolumn{6}{|c|}{1} & \multicolumn{6}{|c|}{2} & \\
\hline Variant & \multicolumn{3}{|c|}{0} & \multicolumn{3}{|c|}{1} & \multicolumn{3}{|c|}{0} & \multicolumn{3}{|c|}{1} & \multicolumn{3}{|c|}{0} & \multicolumn{3}{|c|}{1} \\
\hline Solution no. & 1 & 2 & 3 & 1 & 2 & 3 & 1 & 2 & 3 & 1 & 2 & 3 & 1 & 2 & 3 & 1 & 2 & 3 \\
\hline$\overline{\mathrm{L} \_\mathrm{AT} \text { _S [dB] }}$ & 3.8 & 3.6 & $\mathrm{x}$ & 3.4 & 2.3 & 1.6 & 0.0 & 0.0 & 0.0 & 0.0 & 0.0 & 0.0 & 5.8 & 5.0 & 3.3 & 7.1 & 1.5 & 2.1 \\
\hline $\mathrm{L}_{-} \mathrm{AT}_{-} \mathrm{R}[\mathrm{dB}]$ & 0.1 & 0.2 & $\mathrm{x}$ & 20.0 & 17.9 & 15.9 & 0.0 & 0.0 & 0.0 & 0.0 & 0.0 & 0.0 & 5.3 & 8.1 & 0.9 & 1.4 & 16.5 & 19.9 \\
\hline $\mathrm{T}_{-} \mathrm{D}_{-} \overline{\mathrm{S}}[\mathrm{ms}]$ & 0.0 & 0.0 & $\mathrm{x}$ & 0.0 & 0.0 & 0.0 & 0.5 & 1.5 & 1.2 & 1.9 & 2.4 & 0.0 & 2.7 & 2.6 & 1.8 & 3.3 & 0.1 & 0.4 \\
\hline $\mathrm{T}_{-} \mathrm{D} \_\mathrm{R}[\mathrm{ms}]$ & 0.0 & 0.0 & $\mathrm{x}$ & 0.0 & 0.0 & 0.0 & 0.1 & 0.8 & 0.4 & 3.2 & 3.7 & 0.1 & 0.3 & 0.3 & 0.9 & 0.8 & 3.5 & 3.5 \\
\hline
\end{tabular}


Sound field parameters obtained for the best solutions for $i$-th frequency.

TABLE IV

\begin{tabular}{|c|c|c|c|c|c|c|c|c|c|c|c|c|c|c|c|}
\hline \multirow{2}{*}{ No. } & \multirow{2}{*}{ Variant } & \multicolumn{7}{|c|}{ SPL standard deviation L STD $i[\mathrm{~dB}]$} & \multicolumn{7}{|c|}{ Average SPL on the line L OUT $i$ [dB] } \\
\hline & & 1 & 2 & 3 & 4 & 5 & 6 & 7 & 1 & 2 & 3 & 4 & 5 & 6 & 7 \\
\hline \multirow{2}{*}{ 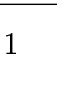 } & 0 & 7.7 & 2.7 & 1.6 & 3.1 & 2.8 & 4.3 & 5.4 & 75.0 & 76.8 & 76.0 & 81.8 & 81.1 & 78.6 & 78.7 \\
\hline & 1 & 3.3 & 2.3 & 4.5 & 4.0 & 3.1 & 3.8 & 4.2 & 80.1 & 78.8 & 76.0 & 79.9 & 77.5 & 77.9 & 78.6 \\
\hline \multirow{2}{*}{2} & 0 & 5.7 & 1.7 & 2.8 & 3.8 & 3.1 & 5.2 & 3.8 & 76.4 & 79.7 & 76.8 & 83.9 & 83.1 & 80.9 & 79.9 \\
\hline & 1 & 4.8 & 4.2 & 3.9 & 7.0 & 5.1 & 6.4 & 4.7 & 82.6 & 81.0 & 78.8 & 82.7 & 81.0 & 81.0 & 81.0 \\
\hline & 0 & 2.4 & 4.4 & 4.1 & 3.8 & 3.8 & 4.4 & 3.4 & 73.0 & 75.5 & 74.9 & 78.9 & 77.4 & 77.6 & 77.2 \\
\hline & 1 & 3.5 & 3.2 & 5.0 & 3.8 & 5.0 & 5.0 & 4.0 & 78.0 & 77.7 & 77.0 & 79.3 & 78.6 & 77.3 & 77.4 \\
\hline
\end{tabular}

\section{Acknowledgments}

This study was supported by the Polish State Committee of Scientific Research (KBN) and the National Scientific Centre under the grant N N504 068538.

\section{References}

[1] W. Ciesielka, PhD Thesis, AGH University of Science and Technology, Kraków 2002, (in Polish).

[2] W. Ciesielka, Archiv. Acoust. 32, 203 (2007).

[3] R. Filipek, PhD Thesis, AGH University of Science and Technology, Kraków 2013, (in Polish).

[4] R. Filipek, A. Gołaś, in: Proc ICSV20 20th International Congress on Sound and Vibration, Bangkok 2013.

[5] A. Gołaś, R. Filipek, Archiv. Acous. 34, 407 (2009).

[6] W. Ciesielka. A. Gołaś, Archiv. Acoust. 31, 89 (2006).

[7] W. Ciesielka, Archiv. Acoust. 34, 559 (2009).
[8] W. Ciesielka, R. Filipek in: Proc. ICSV20, 20th International Congress on Sound and Vibration, Bangkok 2013.

[9] A. Gołaś, K. Suder-Dębska, W. Ciesielka, R. Filipek, Acta Phys. Pol. A 119, 966 (2011).

[10] A. Gołaś, Computer methods for acoustics of interiors and environment, AGH University of Science and Technology Press, Kraków 1995, (in Polish).

[11] A Brański, Analysis of acoustic boundary problems, UR Publishing House, Rzeszów 2001.

[12] T.D. Rossing, Springer Handbook of Acoustics, Springer Science \& Business Media LLC, New York 2007.

[13] I. Harari, Comp. Meth. Appl. Mech. Eng. 195, 1594 (2006).

[14] A. Saltelli, M. Ratto, Global Sensitivity Analysis: The Primer, John Wiley, West Sussex 2008.

[15] ANSYS User Manual, ANSYS Inc., Canonsburg 2013. 